\title{
A comparative analysis of carbon dioxide displacement rates for euthanasia of the ferret
}

\author{
Dawn C. Fitzhugh, VMD, Amanda Parmer, Larry J. Shelton, DVM \& James T. Sheets, DVM
}

Though carbon dioxide asphyxiation is a common method of euthanasia for laboratory animals, species-specific guidelines have not been established for this procedure in the domestic ferret (Mustela putorius furo). The authors investigated the efficacy and stress effects of carbon dioxide euthanasia in 24 ferrets that had participated in previous experimental protocols. They euthanized ferrets by placing them in cages that were either prefilled with carbon dioxide or gradually filled at a displacement rate of $10 \%, 20 \%$ or $50 \%$ of the cage volume per min. Blinded observers subjectively evaluated ferret distress. Prefilling the cage or filling it at a rate of $50 \%$ volume per min resulted in less time to recumbency and to last breath than did filling the cage at a slower displacement rate. Slower carbon dioxide displacement rates also caused an increase in ferret blood glucose concentrations, which may indicate distress. Overall, observers found that prefilling the euthanasia cage caused the least stress to ferrets.

In the annual report of research facilities to the USDA, ferrets are not specifically mentioned but are counted with "all other covered species" 1 . According to one estimate, ferrets probably make up less than $10 \%$ of the animals in this category ${ }^{2}$. Though less common than other laboratory animal species, ferrets have many uses in biomedical research. Some facilities use ferrets to teach pediatric endotracheal intubation techniques because the airway of the ferret resembles the human pediatric airway in size and anatomy ${ }^{3}$. Ferrets are also an important model for the study of human and avian influenza virus transmis$\operatorname{sion}^{4-8}$ and for the development of vaccines against these viruses $^{9-14}$. They are especially useful for studying the synergism of influenza infection and bacterial colonization that occurs in pediatric patients ${ }^{15}$. Additionally, because ferrets are susceptible to infection by the coronavirus that led to the SARS outbreak in 2003 (ref. 16), they may prove to be a useful model for this disease ${ }^{17}$. Ferrets are naturally colonized with Helicobacter mustelae and can present with gastric ulcers, thus providing a model for the attachment and colonization of human Helicobacter pylori ${ }^{18}$ and its role in gastric ulcer formation. The ferret is also a model for human metabolism of carotenoids, which are natural plant pigments ${ }^{19}$.
Many studies involving ferrets require euthanasia to collect tissues and other vital information. In the research environment, carbon dioxide asphyxiation is a common method of euthanasia for many species. Carbon dioxide is inexpensive, and because it is not a controlled substance, it is easily accessible. This euthanasia procedure requires minimal technical proficiency. Many investigators prefer carbon dioxide over other euthanasia methods such as intravenous barbiturates, which may alter parameters under study.

According to the Report of the American Veterinary Medical Association (AVMA) Panel on Euthanasia, carbon dioxide is an acceptable method of euthanasia in small mammals ${ }^{20}$. Though ferrets are not specifically referenced in the report, they are in the same genus as mink (Mustela), which are included. The report states that carbon dioxide is an acceptable method of euthanasia in mink, but indicates that the gas must be administered in high concentrations because mink are burrowing animals and have physiological mechanisms to cope with carbon dioxide exposure. One study showed that mink were able to survive for longer than $15 \mathrm{~min}$ when exposed to carbon dioxide at a concentration of $70 \%$ mixed with $30 \%$ atmospheric air ${ }^{21}$. Because ferrets

Uniformed Services University of the Health Sciences, Bethesda, MD. Correspondence should be addressed to D.C.F. (dfitzhugh@usuhs.mil). 
are burrowing relatives of mink, ferret euthanasia may also require high concentrations of carbon dioxide.

Prefilling the euthanasia cage with carbon dioxide rather than filling the cage when the animal is already inside (gradual fill) can expose the animal to higher concentrations and may lead to a more rapid loss of consciousness and death; however, this method may be distressful to some species ${ }^{22}$. According to the AVMA report, prefilling may be an acceptable method of euthanasia, provided it does not cause distress. Distress resulting from prefilling has not been evaluated in ferrets, and it is not known how prefilling compares with gradual-fill methods for the euthanasia of this species. Additionally, for gradual-fill euthanasia, species-specific guidelines regarding carbon dioxide displacement rates and their effects on the ferret are needed. Though the AVMA report recommends that carbon dioxide should displace at least $20 \%$ of the euthanasia cage volume per minute, this recommendation is based on a rat study ${ }^{23}$ and may not be applicable to ferrets.

In selecting the most appropriate method for euthanasia, potential for distress must be addressed. In humans, within minutes of exposure to almost any physical or neurogenic stressor, the adrenal glands secrete cortisol in response to stimulation by the adrenocorticotropic hormone, often increasing its secretion as much as 20 times $^{24}$. Cortisol then stimulates gluconeogenesis by the liver and decreases cellular use of glucose. The overall effect after a stressful event is a rise in both serum cortisol and blood glucose concentrations. In animals such as the cat, hyperglycemia is a well-documented, rapid, physiological response to stress ${ }^{25,26}$. A previous study of wild ferrets measured glucose concentrations to compare the stress induced by capture using leg-hold traps with that using cage traps ${ }^{27}$. It is unknown whether the domestic ferret experiences a rise in blood glucose in response to stress.

Though a rise in glucose can indicate physiological response to a recent stressor, judgment of an animal's distress is more subjective. One study showed that mink would not enter a chamber prefilled with more than $80 \%$ carbon dioxide by volume ${ }^{28}$. In another study, mink euthanized in a chamber prefilled with carbon dioxide were rapidly rendered unconscious, but the animals convulsed, breathed deeply and emitted whimpering sounds before death ${ }^{21}$. Some observers might associate such behaviors with distress.

Young children, like animals, cannot communicate self-assessment of subjective measures such as distress. There are numerous tools for assisting human pediatric caregivers in observational assessment of these patients. The visual analog scale is one type of rating scale used to determine an observer's impression of pain or other subjective measures in pediatric patients ${ }^{29,30}$. The parent or other proxy rates the subjective measure by drawing a mark on a horizontal line that is $10 \mathrm{~cm}$ long. The $0-\mathrm{cm}$ mark on the line signifies no pain (or other subjective measure), and the $10-\mathrm{cm}$ mark signifies severe pain (or other subjective measure). In the absence of self-reporting capability, observational assessment using rating scales can provide valuable ancillary patient information.

There were several objectives to this study. One was to compare different carbon dioxide displacement rates in terms of time to recumbency and time to last breath (which we used to approximate time to death) in the ferret. Another objective was to determine whether ferrets experienced a rise in blood glucose during euthanasia and if this could provide an objective indication of the differences in stress associated with the displacement rates we tested. The final purpose was to determine, with the help of blinded observers using a visual analog scale, which of the chosen displacement rates was subjectively the least distressful to the ferrets. We collected additional data on the ferrets' behavior during euthanasia to help understand the observers' ratings of distress for each of the displacement rates. To the best of our knowledge, no previous study has assessed behavior, subjective distress or physiological reactions in the ferret in response to carbon dioxide euthanasia.

\section{METHODS}

\section{Ferrets}

We used 24 female domestic ferrets (Mustela putorius furo) ranging in age from 1 to 3 years, weighing 0.65$0.93 \mathrm{~kg}$ each. Mean ferret body weight was $0.78 \mathrm{~kg}$ (s.d. $=0.08 \mathrm{~kg}$ ). All ferrets had been previously vaccinated for rabies and canine distemper and were screened for intestinal parasites by fecal flotation.

As required by previous protocols, ferrets had undergone ovariohysterectomy and anal sacculectomy at least one month before euthanasia. All ferrets received a physical examination by a veterinarian one day before euthanasia. We acquired 23 of the ferrets from Marshall BioResources (North Rose, NY). One ferret was born in our facility under an approved protocol. We group-housed ferrets in enclosures with a total volume of $53 \mathrm{ft}^{3}$. We maintained them on a light:dark cycle of $12 \mathrm{~h}: 12 \mathrm{~h}$ in a temperature-controlled room $\left(64-84^{\circ} \mathrm{F}\right)$. Throughout the study, ferrets had ad libitum access to food (Marshall Premium Ferret Diet, Marshall Pet Products, Wolcott, NY) and water. All ferrets were scheduled for euthanasia after conclusion of approved protocols in which they had participated. All procedures associated with this study were approved by the IACUC in a facility accredited by AAALAC, International.

\section{Euthanasia}

We euthanized each ferret in an amber-colored, clear, clean, polycarbonate rodent cage measuring $42 \mathrm{~cm} \times$ $20.5 \mathrm{~cm} \times 20 \mathrm{~cm}$ (volume $=17.2 \mathrm{l})$. A compressed gas 
cylinder delivered carbon dioxide at a concentration of $100 \%$. A compressed gas regulator (Western Medica, Westlake, $\mathrm{OH}$ ) controlled the pressure of carbon dioxide from the cylinder. A pressure-compensated flowmeter (Western Medica, Westlake, $\mathrm{OH}$ ) regulated the displacement rate. Carbon dioxide entered the euthanasia cage through a stainless steel lid designed for this purpose (Euthanex Corporation, Palmer, PA).

We arbitrarily divided ferrets into four groups. For one group, we prefilled the euthanasia cage with carbon dioxide, which was delivered at a rate of 8.51 (50\% of the cage volume) per min for $3 \mathrm{~min}$. We then placed the ferret in the prefilled cage. In the other three groups, we first placed the ferret in the euthanasia cage and then introduced carbon dioxide at the rate of $1.7,3.5$ or 8.51 per min as indicated on the flowmeter to approximate a displacement rate of $10 \%, 20 \%$ or $50 \%$ of the cage volume per min, respectively. We chose displacement rates of $20 \%$ and $50 \%$ volume per min based on the AVMA panel's global recommendation that at least $20 \%$ of the euthanasia chamber volume be displaced per minute. We euthanized one group in a prefilled cage because burrowing animals such as the ferret may require a higher concentration of carbon dioxide for euthanasia achievable by prefilling. We also included a displacement rate of $10 \%$ volume per min as we hypothesized that the slower rate might provide a slower, gentler death.

We recorded time to recumbency and time to last breath for each ferret. For ferrets euthanized in the prefilled cage, timing began when we closed the lid of the cage after placing the ferret inside. For ferrets euthanized in gradually filled cages, timing began when we turned on the carbon dioxide. We noted recumbency when the ferret made no further attempts to rise and no further attempts to lift its head. We watched ferrets closely for cessation of breathing and recorded the time of last breath. We allowed $1 \mathrm{~min}$ to pass to confirm apnea before turning off the carbon dioxide. We confirmed death by either cardiac auscultation or palpation immediately before or after removing the ferret from the cage.

\section{Subjective evaluation of euthanasia}

Two veterinary technicians observed the euthanasia procedures. Both veterinary technicians had been working as laboratory animal technicians for at least one year each and were experienced and proficient in using carbon dioxide for rodent euthanasia. One technician had assisted in euthanizing one ferret with carbon dioxide a few months before the study. The other technician had never observed the use of carbon dioxide for euthanasia of a ferret. The technicians were blind to the displacement rate of carbon dioxide with the exception of the prefilled cage. For each euthanasia procedure, the technicians received a questionnaire containing seven written questions (Box 1). Some of the questions had corresponding visual analog scales on which the observers marked a number between 0 (no response) and 10 (considerable response). The questionnaire also provided space for the observers to record any comments. The observers did not communicate with each other during the euthanasia.

\section{Blood glucose analysis}

Before placing each ferret in the euthanasia cage, we manually restrained it by holding the scruff of its neck and collected no more than $0.5 \mathrm{ml}$ of blood from the cephalic vein, using a tuberculin syringe with an attached needle. Immediately after euthanasia, we collected no more than $3 \mathrm{ml}$ of cardiac blood percutaneously using a 22-gauge needle on a 3-cc syringe. We used a digital glucometer (OneTouch UltraSmart Blood Glucose Monitoring System, Lifescan, Inc. Milpitas, CA) to analyze glucose concentration in the pre- and posteuthanasia blood samples as soon as they were drawn from the ferret.

\section{Data analysis}

We analyzed all data using SPSS software (SPSS, Inc. Version 12.0 for Windows). We analyzed time to recumbency and time to last breath using one-way ANOVAs with Tukey post hoc analysis. Displacement

\section{BOX 1 D OUESTIONS FOR SUB.]ECTTVE EVALUATTON OF EUTHANASTA}

1. Did this animal attempt to escape from the gas as it filled the chamber? If yes, rate how vigorous the attempt to escape was, on a scale from 0 to 10.

2. Did this animal appear fearful? If yes, rate how fearful the animal was.

3. Did this animal experience signs of respiratory distress such as increased respiratory rate and/or respiratory effort? If yes, rate the degree of respiratory distress.

4. Did this animal appear distressed in any other way (abnormal eye position, dilated pupils, urination, defecation, vocalization)? If yes, rate the degree of distress (other than respiratory).

5. Did this animal experience unexpected side effects during the euthanasia (hemorrhage from nose or mouth, self-trauma, injury, escape, etc.)?

6. Was this an appropriate, efficient method for euthanizing this animal?

7. Please provide any other comments regarding the euthanasia of this animal. 


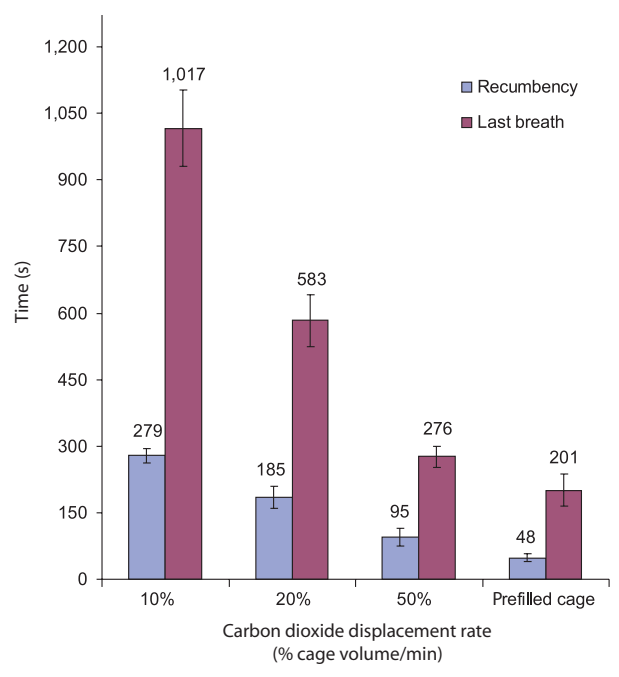

FIGURE 1 | Ferrets' mean time to recumbency and time to last breath from start of euthanasia procedure. Time to recumbency is significantly different between any two groups compared $(P<$ $0.05)$. Time to last breath is also significantly different between any two groups compared $(P<0.05)$, except between the group euthanized in prefilled cages and the group euthanized at a displacement rate of $50 \%$ cage volume per min.

rate was the variable. We analyzed blood glucose with the paired $t$-test $(P<0.05)$.

For each of the four visual analog scales in the questionnaire, we calculated a mean for each group for each observer. We reviewed the observers' written comments and recorded recurring 'themes'. For example, we classified comments such as "no stress," "no suffering" or "no pain" under the theme of "good euthanasia" and reported comments such as "tearing" or "crying" as "lacrimation." If both technicians had similar comments for the same ferret, we counted them only once. We totaled the number of ferrets corresponding to each theme in each euthanasia group. For each group, we also calculated the frequency of affirmative responses to the question, "Was this an appropriate, efficient method for euthanizing this animal?"

\section{RESULTS}

The difference in time to recumbency was significant when comparing between any two euthanasia groups $(P<0.05)$. The difference in time to last breath was also significant between all groups, except between the group euthanized in prefilled cages and the group euthanized at a displacement rate of $50 \%$ of the cage volume per min. Time to last breath was nearly $17 \mathrm{~min}$ in the group euthanized at a rate of $10 \%$ volume per min but less than 3.5 min in the group euthanized in prefilled cages (Fig. 1).

In the group euthanized in prefilled cages, the mean blood glucose concentration after euthanasia was lower than the mean blood glucose concentration before euthanasia. In each of the other groups, blood glucose increased during euthanasia. The rise in blood glucose was statistically significant for groups euthanized at displacement rates of $10 \%$ and $20 \%$ volume per min (Table 1).

The blinded observers' evaluations of the euthanasia methods are summarized in Tables $\mathbf{2}$ and $\mathbf{3}$. Additional responses exhibited by the ferrets that the observers did not document in writing (but discussed with us verbally) included piloerection, head shaking, sneezing, vocalizing and swelling of the jugular region.

\section{DISCUSSION}

It is essential to know how long it takes for animals to be euthanized by various carbon dioxide displacement rates. Knowledge of exposure time is invaluable for programming of automated equipment, and personnel who carry out the procedure should know approximately how long it will take so that they can intervene in a timely manner if equipment is not functioning properly.

In our study, euthanasia using 100\% carbon dioxide occurred in less than $5 \mathrm{~min}$ when cages were filled at higher displacement rates (prefilled cage and gradual fill at $50 \%$ cage volume per min) but took nearly $17 \mathrm{~min}$ at a displacement rate of $10 \%$ volume per min. Ferrets were able to survive for long periods of time when carbon dioxide was slowly introduced into the cage. It is possible that carbon dioxide resistance evolved as a result of the ferret's burrowing behavior. Splenomegaly, a common incidental finding that we have observed in ferrets, may allow for increased oxygen reserve, thus contributing to carbon dioxide resistance.

Ferrets remained in the euthanasia cages for $1 \mathrm{~min}$ after we recorded the time of last breath. We then confirmed death by cardiac palpation or auscultation. This additional minute as well as standard deviations must be included in recommended carbon dioxide exposure times to ensure euthanasia of the ferret at the

\begin{tabular}{lccc} 
TABLE 1 | Mean blood glucose concentrations in ferrets before and after carbon dioxide euthanasia & \\
$\mathbf{C O}_{2}$ displacement rate & $\begin{array}{c}\text { Pre-euthanasia blood } \\
\text { glucose }(\mathbf{m g} / \mathbf{d l})\end{array}$ & $\begin{array}{c}\text { Post-euthanasia blood } \\
\text { glucose }(\mathbf{m g} / \mathbf{d l})\end{array}$ & Difference \\
$\left(\begin{array}{l}\% \\
10 \%\end{array}\right.$ & 77.8 & 106.8 & $29^{*}(P=0.01)$ \\
$20 \%$ & 71.5 & 92.5 & $21^{*}(P=0.01)$ \\
$50 \%$ & 77.7 & 81.2 & $3.5(P=0.40)$ \\
Prefilled cage & 84 & 79 & $-5(P=0.41)$ \\
\hline * Result is statistically significant $(P<0.05)$. & & &
\end{tabular}




\begin{tabular}{|c|c|c|c|c|c|c|c|c|}
\hline \multirow{2}{*}{$\begin{array}{l}\mathrm{CO}_{2} \text { displacement rate } \\
\text { (\% cage volume per min) }\end{array}$} & \multicolumn{2}{|c|}{ Attempted escape } & \multicolumn{2}{|c|}{ Fear } & \multicolumn{2}{|c|}{ Respiratory distress } & \multicolumn{2}{|c|}{ Other distress } \\
\hline & 01 & 02 & 01 & 02 & 01 & 02 & 01 & 02 \\
\hline $10 \%$ & 1.8 & 2.8 & 3.7 & 6.2 & 4.3 & 6.0 & 4.3 & 5.0 \\
\hline $20 \%$ & 0.8 & 0.3 & 2.7 & 4.8 & 3.3 & 6.5 & 2.7 & 5.5 \\
\hline $50 \%$ & 0.3 & 0.2 & 2.6 & 5.2 & 2.5 & 4.8 & 1.3 & 4.3 \\
\hline Prefilled cage & 0.7 & 0.2 & 0.8 & 3.0 & 0.5 & 3.0 & 0.4 & 3.8 \\
\hline
\end{tabular}

various carbon dioxide displacement rates. We note also that only adult female ferrets were included in this study. Future studies should incorporate both sexes and a wider age range to determine whether differences in time to last breath exist between male and female ferrets of different ages.

Animal behaviors during euthanasia can affect personnel and their overall assessment of the animal's level of distress. Many ferret behaviors were consistent for all carbon dioxide displacement rates and included salivation, lacrimation, circling, urination and tremors. Observers also remarked that swelling in the jugular region occurred in nearly all ferrets, though they did not document the frequency. During the time to recumbency, the ferrets had rapid, labored breathing with panting and gasping. When personnel are trained to use carbon dioxide for ferret euthanasia, they should be informed that these responses may occur.

One of the purposes of this study was to determine whether carbon dioxide euthanasia caused changes in blood glucose concentration and whether those changes could provide an objective indication of the stress associated with the different displacement rates. Though all pre- and post-euthanasia blood glucose levels were within normal published reference ranges ${ }^{31}$, there was a statistically significant increase in the blood glucose concentration of ferrets that were euthanized with gradual-fill carbon dioxide at the rates of $10 \%$ and $20 \%$ cage volume per min. The change in blood glucose concentration was not statistically significant for the other two groups. Our study suggests that a statistically significant change in blood glucose concentration can occur during euthanasia when slower fill rates of carbon dioxide are used. Investigators interested in terminal blood glucose levels should euthanize ferrets at a consistent fill rate of carbon dioxide to avoid confounding results.

Though the longer interval between collection of blood samples may account for the increase in blood glucose concentration that occurred with slower filling, this physiological change is consistent with the observers' assessments that slower filling was more distressful to the ferrets (Tables 2 and 3). In ferrets euthanized at a displacement rate of $10 \%$ and $20 \%$ cage volume per min, mean time to recumbency was longer than $3 \mathrm{~min}$. Ferrets seemed conscious and aware of their surroundings and began breathing deeply or gasping for breath as they became recumbent. Neither the $10 \%$ nor the $20 \%$ displacement rate was ever categorized by observers as "good euthanasia." In contrast, the prefilled cage was seen as "good euthanasia" for five of the six ferrets euthanized using this method, and observers categorized the technique as "appropriate" $100 \%$ of the time. Though ferrets exhibited similar behaviors in all groups, the longer time to lateral recumbency caused the observers to determine that slower filling caused greater distress to the ferrets. Consistent with these observations, the rise in blood glucose in ferrets euthanized with slower fill rates is suggestive of a physiological response to a greater degree of neurogenic or physical stress.

In summary, prefilling the euthanasia cage with carbon dioxide resulted in the least amount of time to lateral recumbency and last breath. Observers rated euthanasia in the prefilled cage as the least distressful to the ferrets and their preferred method overall. Slower filling of the cage with carbon dioxide was rated as more distressful to the ferrets and was therefore not as acceptable to the

\begin{tabular}{|c|c|c|c|c|}
\hline $\mathrm{CO}_{2}$ displacement rate ( $\%$ cage volume per min) & $10 \%$ & $20 \%$ & $50 \%$ & Prefilled cage \\
\hline Circling backwards & 1 & 3 & 4 & 0 \\
\hline Salivation & 3 & 3 & 3 & 3 \\
\hline Urination & 0 & 2 & 0 & 3 \\
\hline Lacrimation & 0 & 0 & 2 & 1 \\
\hline Twitching or convulsions & 2 & 1 & 3 & 3 \\
\hline Rapid euthanasia & 1 & 3 & 3 & 3 \\
\hline Prolonged euthanasia & 5 & 3 & 0 & 0 \\
\hline Good euthanasia & 0 & 0 & 3 & 5 \\
\hline Appropriate method & 1 & 3 & 5 & 6 \\
\hline
\end{tabular}


personnel observing the euthanasia. Though blood glucose samples were within reference ranges, slower filling of the euthanasia cage resulted in a statistically significant increase in blood glucose. Investigators should be aware that carbon dioxide displacement rate must be consistent between animals in order to prevent confounding results. Future studies should determine whether additional serum chemistry changes occur at various carbon dioxide displacement rates. They should also include additional objective measures to assess distress, such as cortisol, heart rate, respiratory rate and blood pressure, and should expand the study population to include males and ferrets of different ages.

One purpose of this study was to determine which of the tested carbon dioxide displacement rates provided the least distressful euthanasia. But we would point out that there are alternative euthanasia methods that limit any distress experienced by the ferret. Panting, gasping, piloerection, vocalization, swelling of the jugular region, tremors and salivation occurred even when ferrets were euthanized in a prefilled cage. Euthanasia methods should seek to limit these behaviors as they may be signs of fear and distress in the ferret. Based on our facility's current practices, general anesthesia followed by intravenous or intracardiac injection of barbiturates should be encouraged as the preferred method of euthanasia in the ferret. In facilities that use carbon dioxide, administration of a sedative or anesthetic before introduction to the prefilled cage may also limit the behaviors we observed in this study.

\section{ACKNOWLEDGMENTS}

We acknowledge the dedication of the veterinary technicians who contributed to the many aspects of this project: Romeo Satsatin, Kevin Love and especially Walter Cruz for his insight. We thank the USUHS librarians and biostatisticians for their support.

\section{COMPETING INTERESTS STATEMENT}

The authors declare no competing financial interests.

\section{Received 16 April; accepted 20 August 2007}

Published online at http://www.labanimal.com

1. USDA APHIS. Animal Care Report. (11 December 2007). http:// www.aphis.usda.gov/animal_welfare/downloads/awreports/ awreport2006.pdf.

2. Ball, R.S. Issues to consider for preparing ferrets as research subjects in the laboratory. ILAR J. 47, 348-357 (2006).

3. Powell, D.A., Gonzales, C. \& Gunnels, R.D. Use of the ferret as a model for pediatric endotracheal intubation training. Lab. Anim. Sci. 41, 86-89 (1991).

4. Govorkova, E.A. et al. Lethality to ferrets of H5N1 influenza viruses isolated from humans and poultry in 2004. J. Virol. 79, 2191-2198 (2005).

5. Herlocher, M.L. et al. Influenza viruses resistant to the antiviral drug oseltamivir: transmission studies in ferrets. J. Infect. Dis. 190, 1627-1630 (2004).

6. Maines, T.R. et al. Lack of transmission of H5N1 avian-human reassortant influenza viruses in a ferret model. Proc. Natl. Acad. Sci. USA 103, 12121-12126 (2006).

7. Maher, J.A. \& DeStefano, J. The ferret: an animal model to study influenza virus. Lab Anim. (NY) 33, 50-53 (2004).

8. van Riel, D. et al. $\mathrm{H} 5 \mathrm{~N} 1$ virus attachment to lower respiratory tract. Science 312, 399 (2006).
9. Govorkova, E.A., Webby, R.J., Humberd, J., Seiler, J.P. \& Webster, R.G. Immunization with reverse-genetics-produced H5N1 influenza vaccine protects ferrets against homologous and heterologous challenge. J. Infect. Dis. 194, 159-167 (2006).

10. Hampson, A.W. Ferrets and the challenges of H5N1 vaccine formulation. J. Infect. Dis. 194, 143-145 (2006).

11. Huber, V.C. \& McCullers, J.A. Live attenuated influenza vaccine is safe and immunogenic in immunocompromised ferrets. J. Infect. Dis. 193, 677-684 (2006).

12. Lipatov, A.S., Hoffmann, E., Salomon, R., Yen, H.L. \& Webster, R.G. Cross-protectiveness and immunogenicity of influenza A/Duck/Singapore/3/97(H5) vaccines against infection with A/Vietnam/1203/04(H5N1) virus in ferrets. J. Infect. Dis. 194, 1040-1043 (2006).

13. Mann, A. et al. Interfering vaccine (defective interfering influenza A virus) protects ferrets from influenza, and allows them to develop solid immunity to reinfection. Vaccine 24, 4290-4296 (2006)

14. Suguitan, A.L. et al. Live, attenuated influenza A H5N1 candidate vaccines provide broad cross-protection in mice and ferrets. PLoS Med. 3, e360 (2006).

15. Peltola, V.T., Boyd, K.L., McAuley, J.L., Rehg, J.E. \& McCullers, J.A. Bacterial sinusitis and otitis media following influenza virus infection in ferrets. Infect. Immun. 74, 2562-2567 (2006).

16. Martina, B.E. et al. Virology: SARS virus infection of cats and ferrets. Nature 425, 915 (2003).

17. Subbarao, K. \& Roberts, A. Is there an ideal animal model for SARS? Trends Microbiol. 14, 299-303 (2006).

18. O'Rourke, J.L. \& Lee, A. Animal models of Helicobacter pylori infection and disease. Microbes Infect. 5, 741-748 (2003).

19. Hu, K.Q. et al. The biochemical characterization of ferret carotene- $9^{\prime}, 10^{\prime}$-monooxygenase catalyzing cleavage of carotenoids in vitro and in vivo. J. Biol. Chem. 281, 1932719338 (2006).

20. Beaver, B.V. et al. 2000 Report of the AVMA Panel on Euthanasia. J. Am. Vet. Med. Assoc. 218, 669-696 (2001).

21. Hansen, N.E., Creutzberg, A. \& Simonsen, H.B. Euthanasia of mink (Mustela vison) by means of carbon dioxide $\left(\mathrm{CO}_{2}\right)$, carbon monoxide $(\mathrm{CO})$ and nitrogen $\left(\mathrm{N}_{2}\right)$. Br. Vet. J. 147, 140146(1991).

22. Conlee, K.M., Stephens, M.L., Rowan, A.N. \& King, L.A. Carbon dioxide for euthanasia: concerns regarding pain and distress, with special reference to mice and rats. Lab. Anim. 39, 137-161 (2005).

23. Hornett, T. \& Haynes, A. Comparison of carbon dioxide/air mixture and nitrogen/air mixture for the euthanasia of rodents: design of a system for inhalation euthanasia. Anim. Technol. 35, 93-99 (1984).

24. Guyton, A.C. \& Hall, J.E. Textbook of Medical Physiology 11th edn. (Elsevier Saunders, Philadelphia, 2006).

25. Rand, J.S., Kinnaird, E., Baglioni, A., Blackshaw, J. \& Priest, J. Acute stress hyperglycemia in cats is associated with struggling and increased concentrations of lactate and norepinephrine. $J$. Vet. Intern. Med. 16, 123-132 (2002).

26. Nelson, R. Stress hyperglycemia and diabetes mellitus in cats. J. Vet. Intern. Med. 16, 121-122 (2002).

27. Cross, M.L., Swale, E., Young, G. \& Mackintosh, C. Effect of field capture on the measurement of cellular immune responses in wild ferrets (Mustela furo), vectors of bovine tuberculosis in New Zealand. Vet. Res. 30, 401-410 (1999).

28. Cooper, J., Mason, G. \& Raj, M. Determination of the aversion of farmed mink (Mustela vison) to carbon dioxide. Vet. Rec. 143, 359-361 (1998).

29. Sherman, S.A., Eisen, S., Burwinkle, T.M. \& Varni, J.W. The PedsQL Present Functioning Visual Analogue Scales: preliminary reliability and validity. Health Qual. Life Outcomes 4 (2006).

30. von Baeyer, C.L. \& Spagrud, L.J. Systematic review of observational (behavioral) measures of pain for children and adolescents aged 3 to 18 years. Pain 127, 140-150 (2007).

31. Quesenberry, K.E. \& Carpenter, J.W. Ferrets, Rabbits, and Rodents Clinical Medicine and Surgery 2nd edn. (Saunders, St. Louis, 2004). 